\begin{tabular}{|c|c|}
\hline 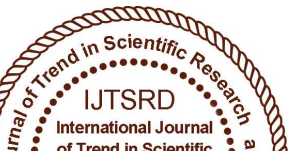 & $\begin{array}{l}\text { International Journal of Trend in Scientific } \\
\text { Research and Development (IJTSRD) }\end{array}$ \\
\hline $\begin{array}{lll} & \\
0\end{array}$ & International Open Access Journal \\
\hline 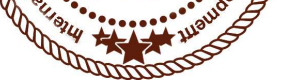 & ISSN No: $2456-6470 \mid$ www.ijtsrd.com | Volume - 2 | Issue -5 \\
\hline
\end{tabular}

\title{
A Survey on Classification and Prediction Techniques in Data Mining for Diabetes Mellitus
}

\author{
T. Padma Nivethitha ${ }^{1}$, M. Uma Maheswari ${ }^{1}$, Dr. J. G. R. Sathiaseelan ${ }^{2}$ \\ ${ }^{1}$ Research Scholar, ${ }^{2} \mathrm{Head} \&$ Associate Professor \\ Department of Computer Science, Bishop Heber College \\ Trichy, Tamil Nadu, India
}

\begin{abstract}
The medical industry incredibly utilizes the data mining systems for different expectations and characterization. The substantial data repositories produced is subjected to different calculations to distinguish the examples in the data. The diabetic is the most undermining ailment with the end goal where millions of people suffers each year. In this paper the forecast of the diabetics is done by utilizing different procedures like classification and prediction techniques decision tree, Naive Bayes, Support vendor machine(SVM), clustering, K-Nearest Neighbour, K-means, K-medoids, Neural Networks, Association rule mining and Multilayer Preceptron have been examined broadly. It is seen from the examination that the Naĩve Bayes and C4.5 algorithm system show to have better execution with satisfactory results.
\end{abstract}

Keywords: Data Mining, Diabetes, Prediction, C4.5, Naïve Bayes.

\section{INTRODUCTION}

Data mining is an expansive zone that incorporates procedures from a few fields including machine learning, statistics, pattern recognition, artificial intelligence, and database systems, for the examination of vast volumes of data. There have been an extensive number of data mining algorithms attached in these fields to perform diverse data examination assignments. Data Mining is the way toward extracting covered learning from huge volumes of rough data. The learning must be new, not clear, and one must have the capacity to utilize it.
Data mining has been de-fined as "the nontrivial extraction of already unclear, certain and possibly valuable data from data. It is "the exploration of

extricate helpful data from vast databases". It is one of the shops during the time spent learning revelation from the database. Data Mining is utilized to find learning Out of data and displaying it in a shape that is effectively comprehended to people. Now a days expire forecast assumes an imperative part in data mining. There are distinctive kinds of expire anticipating in data mining to be specific cardiac disease, lung cancer, breast cancer and diabetic. Diabetes mellitus now turned into a ssignificant medical issue. Diabetes is a expire where the body couldn't deliver insulin or not utilizing created insulin accurately. The insulin subordinate diabetes mellitus (IDDM) is a chronic disease that shows up when hormone insulin has not been created enough in patients a body. This expire in expanding step by step as indicated by International diabetes Federation, there right now 246 million diabetic individuals around the world, and this number is required to ascend to 380 million by 2025 . Health data mining is a test to include a misdiagnosis and hesitation. The following diagram depicts the process in medical data mining.

\section{TECHNIQUES USED IN DATA MINING:}

Data disclosure in therapeutic data is the way toward extricating distinctive highlights from data in different advances. Fig.1 [1] demonstrates the procedure of System Architecture 


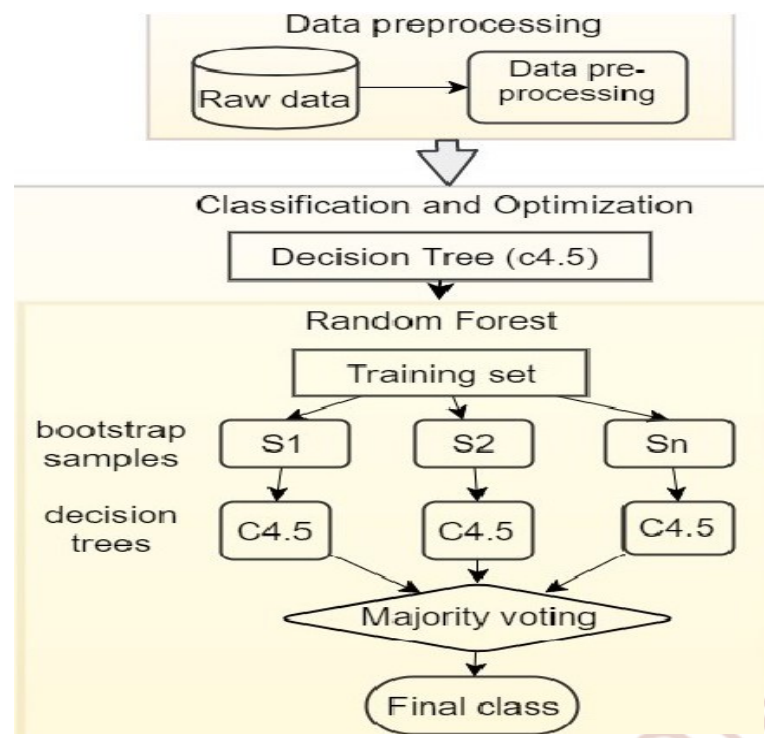

Figure1: System Architecture.

\section{Classification:}

Classification is a machine learning based data mining procedure. Order is utilized to characterize every data in an arrangement of data into one of predefined set of gatherings or classes. It makes utilize scientific methods, for example, decision trees, direct programming, neural networks and insights to order the data into various gatherings. In the recent past, the classification strategies give more clever techniques to absorbing expectation of diseases. Diverse sorts of classification methods incorporates Support vector machine, discriminate investigation, naive Bayes, decision trees, linear and non-linear regression.

\subsection{Decision Tree:}

Decision tree is a tree structure, which is as a flowchart. It is utilized as a technique for combination and probability with exposé utilizing hubs and sub hubs. The root and inner hubs are the experiments that are utilized to isolate the examples with various highlights. Inside hubs the mselves are the consequence of quality experiments. Leaf hubs mean the class variable. Decision tree gives an effective procedure to arrangement and forecast in Diabetes conclusion issue. Every hub for the Decision tree is found by establishing the most significant data pick up for all characteristics and if a particular quality gives an explicit finished result (clear characterization of class property), the branch of this characteristic is ended and target respect is allotted to it. Fig.2[2]. Demonstrates an example decision tree structure.

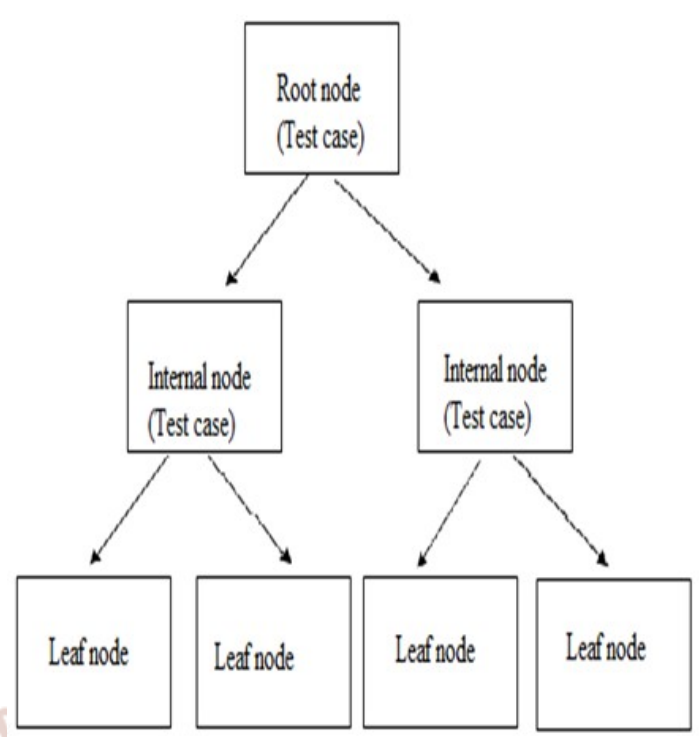

Figure 2.Sample Decision Tree Structure

\subsection{Nä̈ve Bayes:}

In medicinal data mining, Naive Bayes grouping assumes an essential part. It is a probabilistic order in view of the Bayes theorem.

"Naive" suggests the freedom between all qualities. Naïve Bayes (NB) is a machine-learning technique that has been utilized for more than 50 years in biomedical informatics. It requires just little measure of preparing data to assess the parameter which is exceptionally helpful for human services applications. Bayes order is delayed by current methodologies, as supported trees or irregular timberlands.

\subsection{C4.5 Algorithm:}

In Classification strategies, $\mathrm{C} 4.5$ algorithm is utilized to create choice tree. Upgrades from ID.3 algorithm is C4.5 algorithm. Utilizing the idea of data entropy, C4.5 manufactures choice trees from an arrangement of preparing data. C4.5 takes after a post pruning approach. Data pick up is standardized from the part criteria. Utilizing partition and defeat algorithm, C4.5 first grows an underlying tree. This algorithm performs well in commotion free data.

Advantage:

$>$ C4.5 algorithm develop trees and develop its branches.

$>$ The quality with the most significant standardized data pick up is settled on the choice.

$>$ This algorithm is utilized to deal with constant and discrete qualities. 
Disadvantage:

$>$ The $\mathrm{C} 4.5$ algorithm contains exhaust branches.

$>$ The irrelevant branches not just lessen the ease of use of choice.

D Over fitting occurs in C4.5 algorithm.

\subsection{Support Vector Machine:}

The principle point of support vector machine is to locate the exact characterization strategy to separate between individuals from the two classes in the preparation data. In the support vector machine strategy the ideal limit is known as hyper plane. The vectors that are set close to the hyper plane is called supporting vectors. In the event that the space isn't straightly distinguishable there might be no isolating hyper plane.

Advantage:

$>$ Support vector machine can be effectively reached out to perform numerical counts.

$>$ Support vector machine is extremely helpful for general example recognition, relapse and order. Support vector machine can be utilized for design order which has multilayer observations and outspread premise work organize.

Disadvantage:

$>$ Support vector machine is computational wastefulness.

\section{Neural Network:}

In practical applications, neural networks are prominent to make outstandingly exact results. By using neural network, variable learning rate and back propagation learning calculation with liveliness, the neural networks is set up with diabetes data set. The layout of the model is according to the accompanying: It starts with the commitment of clinical data and advances to make ANN figuring. In the wake of planning model, it can make the desire comes out. The computational steps of neural networks estimation begin with the description of clinical data into two proportional measures of randomly. One is used for testing and the other is used for preparing. A hidden weight is allotted to every component discretionarily. The figured errors are used to change the greatness of all features.

\section{Multilayer perceptron neural network (MLPNN):} Multilayer perceptron neural systems (MLPNNs) are the most regularly utilized provide for forward neural systems because of their fast operation, simplicity of usage, and set supplies. The MLPNN comprises of three consecutive layers: input, covered up and yield layers. The covered layer forms and transmits the info data to the yield layer. A MLPNN show with lacking or unreasonable number of neurons in the covered layer in all probability causes the issues of poor assumption and over fitting. There is no investigative technique for determining the number of neurons in the covered layer.

\section{Clustering:}

Clustering is a data mining system that makes important or accommodating group of substance that have comparable component utilizing mechanical strategy. Divergent from grouping, clustering procedure additionally characterizes the classes and place questions in them, as in characterization objects are allotted into predefined classes. For instance in forecast of coronary illness by utilizing clustering get group or express that rundown of patients which have same hazard factor. Assets this influences the split rundown of patients with high blood to sugar and related hazard factor so on.

\subsection{K-means:}

K-Means is an unverified Clustering method for commonly sectioning an educational accumulation into $\mathrm{k}$ gatherings. In this procedure, $\mathrm{k}$ address different gatherings, it is typically a customer push to the methodology; some model can be used to therefore assess $\mathrm{K}$. The mean estimation of the constituent is full beyond what many would consider possible to design Clusters. The groups mean or center is to be made by the random decision. For each vector, this estimation figures the detachment between data vectors promote more by each gathering centroid using the current shape.

\subsection{K-medoids:}

K-Medoid algorithms are familiar with discovering Medoid in a collection which is center position hub in a gathering. The essential arrangement of a K-Medoid aggregate estimation is to find clusters in $\mathrm{N}$ challenges by first discretionarily finding a delegate question for each gathering. The each parallel inquiry is gathered with the Medoid. It uses the delegate objects are reference hub as opposed to taking the mean estimation of the parts in each group. The count goes before the data factor of $\mathrm{k}$, and the quantity of gatherings to be allocated a game plan of $\mathrm{N}$ objects. Therefore, K-Medoid is more stranded when appeared differently in relation to K-Means. 


\subsection{K-nearest neighbour algorithm:}

$\mathrm{K}$-nearest neighbour is a managed learning calculation created, where the consequence of new case question is arranged in light of dominant part of K-nearest neighbour class. The motivation behind this calculation is to characterize another protest in light of memory. Given an inquiry point, we discover $\mathrm{k}$ number of items or (preparing focuses) nearest to the question point. The characterization is utilizing larger part among the order of the k-objects. Any ties can be broken aimlessly. $\mathrm{K}$ nearest neighbour calculation utilized neighbourhood arrangement as the forecast estimation of the new question case. $\mathrm{K}$ nearest neighbour calculation is exceptionally basic.

\section{Association:}

Association is frequently used data mining techniques as compared to other known data mining system. In association, an example is uncovered in light of a relationship of a specific thing on different things in a similar task. For instance, the association procedure is utilized as a part of diabetics illness expectation as it say to us the relationship of unique characteristics utilized for investigation and deal with the patient with all the hazard factor which are essential for forecast of malady.

\section{LITREATURE SURVEY}

Sathya et al. [3] proposed in this paper the two data mining calculations Random tree and ID3 were actualized with the diabetic dataset with 13 traits and 642 occurrences. The usage has been finished with the famous data mining instrument Weka. It is discovered that Random tree yields the precision of $94.7867 \%$ and ID3 gives the exactness of $96.3665 \%$. The proposed half breed display joins the highlights of Random Tree and ID3 calculation and produces the enhanced exactness of $99.0521 \%$. This paper presumes that Hybrid model produces enhanced rate of precision to anticipate the diabetic.

Tejashri et al.[4] proposed that it was accounted for that $\mathrm{J} 48(\mathrm{C} 4.5)$ had defeated over different methods by demonstrating $100 \%$ exactness. J48 is extremely straightforward and exact classifier to settle on a choice tree over different classifiers. The outcome got by utilizing managed machine learning had demonstrated that the time taken for data investigation was high in KNN. The exactness was high and time taken was minimum in J48 (C4.5). This demonstrates the computational cost for data examination was low in $\mathrm{J} 48$ (C4.5) thus the execution is precise.
Senthil Kumar et al. [5] proposed that the clinical movement examination assumes a vital part in current pattern. Discovery and examination of clinical movement is the most imperative issue continuously situation, in light of the fact that the absence of preparing tests and adequate data's make these procedures much confounded. This clinical data examination can be performed by successful data mining systems and methodologies. There are a few distinct techniques to analysis and anticipation diabetes mellitus. This review introduces a different strategies of the data mining way to deal with take care of the diabetes ailment finding issue. From the examination we find a few issues and finds in clinical datasets taking care of process.

Saman Hina et al. [6] proposed to make successful and effective outcomes, the prerequisite is to chip away at various calculation and to ensure which suits best. Diagnosing diabetes through data mining apparatus over therapeutic records of patients however it has been finished by a larger part of the specialists [10-15] yet the exploration requests all the more profound delving as far as area learning to get more agent restorative analysis. Regarding execution, it was discovered that multilayer discernment work is best subsequently it indicates less blunders anyway it requires excessively handling investment since it requires estimation of weights of every hub. ZeroR is valuable to decide pattern execution for others order strategy. Guileless Bayes is likewise extremely productive as it gives an overwhelming outcome after every approval except its execution isn't stopped amazing. J4.8 gives a graphical picture of the priority of the trait as it ascertains the need of each property with other but then it additionally predicts exact outcomes with slightest mistake subsequently it requires time. The target of looking at the calculation on the same dataset, breaking down and foreseeing the outcomes out of it has been accomplished. In future, creators are occupied with social event data among our own particular neighbourhood and creators were quick to get new outcomes which lead them toward more exact and precise divination.

Manimaran et al. [7] proposed Five data mining order systems were looked at on numerous elements on a similar arrangement of properties in the MV database. They comes about were gotten for MLP, Bayes Net, JRip, FLP, and C4.5 characterization strategies. The methods were thought about on time, exactness, review and mistake rate. It was discovered that 
BayesNet, MLP, FLP had bring down calculation time. On exactness, C4.5 and JRip had precision over $85 \%$. Accordingly this work reasons that $\mathrm{C} 4.5$ and JRip are the most suited calculations for expectation utilizing order on datasets with infected kidney patients. Therapeutic forecasts require higher precision levels and exactness over $85 \%$ is useful for early identification/expectation of diabetes, in this way helping specialists take preventive and early activities on treatment.

Suresh Kumar et al. [8] proposed that the diabetes is most normally happening infection. Forestalling, controlling and making mindfulness about diabetes is critical as it prompts other medical issues. Sort 1 and sort 2 diabetes may prompt heart issues, kidney diseases and eye related problems. It is imperative to anticipate or control gestational diabetes in light of the fact that Gestational Diabetes Mellitus (GDM) may leave after pregnancy, yet ladies who have GDM seven times more are probably going to create write 2 diabetes than ladies who don't have GDM in pregnancy. The offspring of the GDM mother have the danger of heftiness and sort 2 diabetes. These challenges can be dealt with by controlling the glucose levels. From this investigation, it / was discovered that data mining strategies can be utilized for foreseeing the sort and hazard levels of diabetes. Through this examination it is discovered that the data mining methods are essential and it prompts substantial methodologies for anticipating the danger of gestational diabetes. So it is our proposal to utilize new methods like data digging for basic leadership in therapeutic fields, which enhances the analysis of ailments like gestational diabetes. This examination helps the specialists and wellbeing associations in utilizing the data mining methods in the medicinal field which helps in anticipating the kind of diabetics and dangers levels related with it. Accordingly the proposed show helps in enhancing the determination of the sicknesses which in reality helps in early cure of infection in the patients.

Selvakumar et al. [9] proposed this work centred the usage of Binary Logistic Regression, Multilayer Perceptron and k-Nearest Neighbour for the diabetes data. From the examination, it is analyzed that the development of groupings will be diverse for arrangement strategies. From the histogram, it is seen that the Binary Logistic Regression exactness is 0.69, Multilayer Perceptron precision is 0.71 and $\mathrm{KNN}$ gives the exactness of $0.80 . \mathrm{kNearest}$ Neighbour is higher than the exactness of Binary Logistic Regression and Multilayer Perceptron.

Haoting Zhang et al. [10] proposed the consequence of relationship, we discovered that release aura and confirmation compose had the most elevated connection esteem. Be that as it may, these two highlights were not firmly connected to the infection itself, but rather connected to medicinal administration. In this way, we concocted a conclusion: Medical administration is critical in expanding patients' likelihood of not being readmitted. What sort of condition does the clinic give to the patient amid and after the treatment (e.g. released to home or another fleeting clinic, or to home with home wellbeing administration, or to a long haul mind healing facility, and so on.) is as vital, if not all the more telling, as the treatment itself. In this investigation, we discover that utilizing order models in Rapid Miner can give us a noteworthy expectation of whether patients will be readmitted inside 30 days, by essentially contributing the patients' data into the choice tree model and running it. The model would itself be able to figure an outcome. In the event that the outcome is "Awful", which implies the patient is probably going to be readmitted inside 30 days, specialists and attendants can endeavour to alter the highlights of the patients (time in doctor's facility, number of lab strategies, number of methodology, number of solutions, glucose serum test, $\mathrm{HbAlc}$ test, change in medicines, and diabetes drugs). Subsequent to customizing the treatment, the model can be run by and by to see whether the outcome is changed to "Great". In the event that it is changed, at that point the balanced data can be the new arrangement of treatment for the patient. In the wake of treating the patient in a way which makes his/her practical data coordinated to the balanced one, he/she will have a lower probability of being readmitted. Additionally, the significance of therapeutic administration is accentuated. The place that the patient stays completes a great deal to the treatment impact, as indicated by the relationship result. Thusly, giving patients an appropriate domain to remain in is critical, and can change the after-effect of their treatment. Our future work will investigate the likelihood of building up a prescriptive advancement technique with the goal that the program can locate the best customized method for treating diabetes patients without anyone else, which implies it will never again be vital for people to be engaged with customizing the treatment. 
Harnoorkapur et al.[11] proposed about 10 years back for upgrades in the fields of machine learning and data mining. The utilization of these procedures in medicinal field assume a critical part in sickness grouping and expectation. Considering the growing mortality of patient experiencing diabetes consistently, researchers are using data mining procedures in the examination of the beginning of diabetes. Indeed, even with the acknowledgment that using data mining methodologies to help medicinal services specialists in the investigation of diabetes has certain accomplishment, the usage of data mining frameworks to perceive a fitting treatment for patients experiencing this sickness has gotten little thought. This paper shows a one of a kind ensembled approach with multi-target greater part voting method connected on diabetes dataset to enhance the sickness forecast and arrangement precision. Future research bearings may incorporate outfit which can incorporate its execution on numerous more illnesses like malignancy and cardiovascular issue for expectation and characterization purposes. The framework may be adjusted by the utilization of other amazing gathering methods like Dagging, Stacking, Rotation Forest, Decorate, and so forth.

Ehsanpashaee et al. [12] proposed as per our outcomes ladies in the age scope of more seasoned than 80 who are heavier than $75 \mathrm{KG}$ and men in the age scope of more established than 70 who are heavier than $75 \mathrm{KG}$ are at high hazard for creating blood circulatory and diabetic issue. We have discovered that this age gathering would as a rule look for the assistance of a general professional to treat their difficulties and side effects; the most noteworthy rate of insulin utilize was likewise among this age gathering. To be more particular, we can reason that men are at higher hazard for creating diabetes than ladies. We have decided the primary driver of diabetes illness to be either inertia because of maturity or additional weight. At long last, we infer that inability to look for treatment for diabetes may exacerbate this condition and may prompt heart and coronary or kidney illnesses.

Ravi sanakal et al. [13] proposed that the early identification of any sort of sickness is a fundamental factor. This aides in treating the patient well ahead. In this, examine paper is intended to outline a framework that would help specialists in therapeutic finding. This paper displays a demonstrative FCM and in addition SVM utilizing SMO and chooses which system helps in determination of Diabetes ailment. The best outcome is by FCM with a precision of $94.3 \%$ and positive prescient esteem which is $88.57 \%$. SVM has a precision of $59.5 \%$ which is very low. These outcomes are very tasteful, because of the way that identifying the Diabetes is an exceptionally complex issue. Maybe the most imperative after-effect of this investigation was the understanding increased through the usage and the outcomes acquired here are additionally extremely reassuring and open the entryways without bounds inquire about towards the location of Diabetes infection. This examination can be additionally stretched out to bargain datasets with various classes.

Rajesh et al. [14] proposed they have connected numerous order calculations on Diabetes dataset and the execution of those calculations have been examined. An order rate of $91 \%$ was gotten for $\mathrm{C} 4.5$ calculation. Future upgrade of this work incorporates spontaneous creation of the $\mathrm{C} 4.5$ calculations to enhance the grouping rate to accomplish more prominent precision in order.

Brindha et al.[15] proposed the test considers, the dataset have been apportioned into $52 \%$ preparing and $46 \%$ for testing of KNN and ID3 calculation. It has been performed on PIDD and the outcomes are looked at. It might be seen that by applying the Feature Selection strategy, 7 traits have been chosen from 8 characteristics and by performed arrangement of the chose qualities. The proposed strategy ID3 classifier boosts the arrangement precision thsn the current technique KNN. For the future research work, we proposed to create master framework with various Feature Selection and Classification strategies which could fundamentally diminish human services cost by means of early expectation and conclusion of diabetes.

Krishnaveni et al. [16] proposed that the naives Bayes is more gainful than different classifiers. Consequently this article presents an effective Diabetic mellitus Diagnosing method which predicts the infection that can at last diminishing the manual work. We started with watching the side effects as it are exceptionally hard to anticipate diabetes mellitus perish discovering indications. In the second step we preprocess the diabetic database to influence the mining to process more productive. At last, the outcomes are contrasted and the assistance of various expectation classifiers Discriminent examination, 
KNN, Naïve Bayes and Support vector machine. The last outcomes were thought about utilizing distinctive execution measures. These measure utilized genuine positive (TP), genuine negative (TN), false positive (FP) and false negative (FN) to figure comes about. Exhibitions of our system were estimated by Accuracy: 74.1155\%. The proposed approach has exhibited that mining recovers valuable relationship even from properties which are not immediate pointers of the class we are endeavoring to anticipate. Other than these data investigation results can be used for additionally look into as a piece of updating the precision of the forecast framework in future.

Vrushali et al. [17] proposed the Amount of Research work has been improved the situation Prediction of diabetes utilizing data mining strategy. The base up rundown method utilizes when tolerant has high danger of diabetes. The K-Nearest Neighbour Algorithm, Bayesian Classifier, Naïve Bayesian Classifier, Artificial Neural Network, Bayesian Network, Association Rule Mining all strategies utilized for forecast of diabetes which gives patients state of Normal, Pre-diabetes, Diabetes. In K-Nearest neighbour calculation dependably need to decide the estimation of K. Every single above strategy used to anticipate diabetes. Be that as it may, if Patient is recognized as diabetes initially there is a need of discovering Control and Un-control state of diabetes. Since if Patient has diabetes in Un-control condition, might be the patient has extreme impact on Patient's Organ like Heart, Eye, Kidney and so on. So there is need of finding early Severity which might be help tolerant for lessening the Severity on Organ or Halting the Severe Effect on Organ.

Harleen et al. [18] proposed the general target of the data mining system is to focus data from a data set and change it into a sensible structure for additionally use. Close to the rough examination step, it incorporates database and data organization points of view, data pre-handling, model and enlistment thoughts, intriguing quality estimations, multifaceted design considerations, post-getting ready of discovered structures, representation, and web overhauling. Different order approaches had been actualized in data mining process. These methodologies have been utilized to isolate the data into various sets so effectively connection between various characteristics can be recognized. Diverse data mining procedures have been utilized to enable wellbeing to mind experts in the conclusion of
Diabetes illness. Those most often utilized spotlight on arrangement: gullible bayes choice tree, and neural system. Other data mining procedures are likewise utilized including part thickness, consequently characterized gatherings, sacking calculation, and bolster vector machine. Though applying data mining is beneficial to health care, disease diagnosis, and treatment, few looks into have examined creating treatment gets ready for patients. The principle issue in the diabetes data classification is that due to insufficient resources and data proper mining has not been done.

Monika et al. [19] proposed the medicinal machine learning has picked up in enthusiasm by the logical and research groups. Diabetes is considered as the world's quickest developing constant ailment. It needs ceaseless self-administration and control to keep up blood glucose level inside the typical range, so as to avoid confusions and forestall diabetic occasions. Diabetic is a condition that happens when blood glucose is too low. The event of diabetic may bring about seizures, obviousness, and conceivably changeless mind harm or demise. They also proposed a model in foreseeing diabetes by applying data mining system. Diabetes mellitus is a perpetual sickness and a noteworthy general wellbeing challenge around the world. Utilizing data mining techniques to help individuals to anticipate diabetes has increase significant fame. In this Bayesian Network classifier is proposed to foresee the people whether diabetic or not. Results have been acquired.

Divya et al.[20] proposed the goal of our work is to give an investigation of various data mining procedures that can be utilized in robotized way of life ailments forecast frameworks. Different methods and data mining classifiers are characterized in this work which has developed as of late for productive and viable coronary illness and sort II diabetes finding. This investigation demonstrates that diverse innovations are utilized with various number of properties. Along these lines, distinctive innovations utilized demonstrated the diverse exactness to each other. In some coronary illness papers it is demonstrated that neural system given the exactness of $100 \%$ while choice tree and innocent bayes gives $99.0741 \%$ and $99.52 \%$ precision individually. Furthermore, in some diabetes papers it is demonstrated that MLP gives the precision of $99.10 \%$ , SVM gives $97.98 \%$ and Naïve Bayes gives $95 \%$ of exactness. Along these lines, distinctive advances 
utilized demonstrated the diverse precision relies on number of characteristics taken and apparatus utilized for usage. The accessibility of enormous of sum and overall expanding mortality of way of life infections, analysts are utilizing data mining procedures in the conclusion of coronary illness and sort II Diabetes. In spite of the fact that applying data mining strategies to help social insurance experts in the determination of way of life malady is having some achievement, the utilization of data mining procedures to recognize a reasonable treatment for way of life ailment patients has gotten less consideration.

\section{TABLE 1: DIAGNOSIS OF DIABETICS USED DIFFERENT DATA MINING TECHNIQUES}

\begin{tabular}{|c|c|c|c|}
\hline $\begin{array}{l}\text { S. } \\
\text { No }\end{array}$ & Author & Techniques & Acuracy \\
\hline 1 & Sathya et al. & $\begin{array}{l}\text { Random Tree } \\
\text { Naïve Bayes }\end{array}$ & $\begin{array}{l}94.79 \% \\
96.37 \%\end{array}$ \\
\hline 2 & Tejashri et al. & $\begin{array}{l}\text { Naïve Bayes } \\
\text { C4.5 } \\
\text { Decision Tree } \\
\text { Neural } \\
\text { Networks }\end{array}$ & $\begin{array}{l}98.85 \% \\
98 \% \\
98.48 \% \\
97.85 \% \\
9\end{array}$ \\
\hline 3 & $\begin{array}{l}\text { Senthilkumar et } \\
\text { al. }\end{array}$ & $\begin{array}{l}\text { Decision Tree } \\
\text { Naïve Bayes }\end{array}$ & $\begin{array}{l}85.090 \% \\
81.010 \%\end{array}$ \\
\hline 4 & SamanHina et al. & $\begin{array}{l}\text { Naïve Bayes } \\
\text { MLP }\end{array}$ & $\begin{array}{l}76.3 \% \\
81.8182 \%\end{array}$ \\
\hline 5 & Manimaran et al. & $\begin{array}{l}\text { MLP } \\
\text { Bayes Net } \\
\text { JRIP } \\
\text { C4.5 }\end{array}$ & $\begin{array}{l}75 \% \text { eve } \\
85 \% \\
86 \% \\
86 \%\end{array}$ \\
\hline 6 & $\begin{array}{l}\text { Sureshkumar et } \\
\text { al. }\end{array}$ & $\begin{array}{l}\text { Navie Bayes } \\
\text { Random Tree } \\
\text { C4.5 }\end{array}$ & $\begin{array}{l}90.9 \% \\
96.3 \% \\
98 \% \\
\end{array}$ \\
\hline 7 & $\begin{array}{l}\text { Selvakumar et } \\
\text { al. }\end{array}$ & $\begin{array}{l}\text { MLP } \\
\text { Binary } \\
\text { Logistic } \\
\text { Regression } \\
\text { K-Nearest } \\
\text { Neighbour }\end{array}$ & $\begin{array}{l}71 \% \\
69 \% \\
80 \%\end{array}$ \\
\hline 8 & $\begin{array}{l}\text { Haoting Zhang } \\
\text { et al. }\end{array}$ & $\begin{array}{l}\text { Rule } \\
\text { Induction } \\
\text { Decision Tree } \\
\text { Navie Bayes } \\
\end{array}$ & $\begin{array}{l}77 \% \\
75.50 \% \\
75.17 \%\end{array}$ \\
\hline 9 & $\begin{array}{l}\text { HarnoorKapur et } \\
\text { al. }\end{array}$ & $\begin{array}{l}\text { SVM } \\
\text { Decision Tree }\end{array}$ & $\begin{array}{l}74.32 \% \\
76.19 \% \\
\end{array}$ \\
\hline 10 & $\begin{array}{l}\text { EhsanPashaee et } \\
\text { al. }\end{array}$ & $\begin{array}{l}\text { Naïve Bayes } \\
\text { Bayes Net } \\
\text { Random } \\
\text { Forest }\end{array}$ & $\begin{array}{l}80.29 \% \\
80.83 \% \\
99.63 \%\end{array}$ \\
\hline
\end{tabular}

\section{Accuracy}

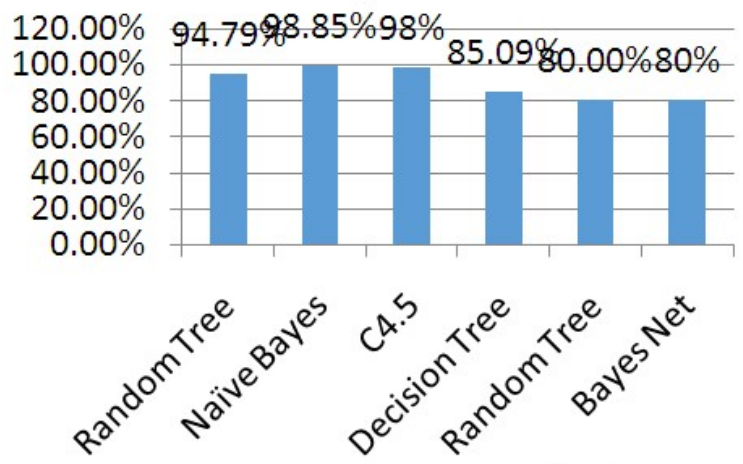

Figure.3: Graphical representation of the data mining techniques reviewed

\section{CONCLUSION}

On looking at different data mining methods for forecast of diabetics, C4.5 algorithm and Naïve Bayes indicates more accuracy than different strategies. The accompanying table has been seen from the broad examination on the calculations in the expectation of the diabetes ailments. This review gives an elaborate examination on the procedures, methodologies, qualities and undersized comings. This elaborate think about gives suggestions to the analysts and specialist to work together to perform straightforward clinical data sets for the data mining models.

\section{REFERENCES}

1. Procheta Nag, SaikatMondal, Foysal Ahmed," A Simple Acute Myocardial Infarction (Heart Attack) Prediction System Using Clinical Data and Data Mining Techniques",2017 20th International Conference of Computer and Information Technology (ICCIT), 22-24 December, 2017, 978-1-5386-1150-0/17/\$31.00c 2017 IEEE.

2. AiswaryaIyer, S. Jeyalatha and RonakSumbaly," Diagnosis of Diabetes Using Classification Mining Techniques", International Journal of Data Mining \& Knowledge Management Process (IJDKP) Vol.5, No.1, January 2015 DOI.

3. S. Sathya, A. Rajesh and K. Bhuvaneswari," Hybrid Data Mining Techniques For Accurate Diabetic Prediction", I J C T A, 9(28) 2016, pp. 301-306C International Science Press.

4. Ms.Tejashri N. Giri, 2prof. S. R. Todamal," Data Mining Approach For Diagnosing Type 2 Diabetes", ISSN: 2348-4098 VOL 2 ISSUE 8 NOV-DEC 2014.

5. B. Senthil Kumar, Dr. R. Gunavathi," A Survey on Data Mining Approaches to Diabetes Disease 
International Journal of Trend in Scientific Research and Development (IJTSRD) ISSN: 2456-6470

Diagnosis and Prognosis", International Journal of Advanced Research in Computer and Communication Engineering ISO 3297:2007 CertifiedVol. 5, Issue 12, December 2016, ISSN (Online) 2278-1021 ISSN (Print) 23195940.

6. SamanHina, Anita Shaikh and SohailAbulSattar," Analyzing Diabetes Datasets Using Data Mining", Journal of Basic \& Applied Sciences, 2017, 13, 466-471.

7. R. Manimaran and Dr. M. Vanitha,'Prediction of Diabetes Disease Using Classification Data Mining Techniques", International Journal of Engineering and Technology (IJET), ISSN (Print): 2319-8613 ISSN (Online): 0975-4024.

8. P. Suresh Kumar And V. Umatejaswi,"Diagnosing Diabetes using Data Mining Techniques", International Journal of Scientific and Research Publications, Volume 7, Issue 6, June 20175 ISSN 2250-3153.

9. S.Selvakumar,K.SenthamaraiKannan,S.GothaiNac hiyar,'Prediction of Diabetes Diagnosis Using Classification Based Data Mining Techniques", International Journal of Statistics and Systems ISSN 0973-2675 Volume 12, Number 2 (2017), pp. 183-188 (C) Research India Publications.

10. Haoting Zhang, Lingyun Shao, Chengyu Xi," Predicting the treatment effect in diabetes patients using classification models",

11. HarnoorKaur, ShaliniBatra," HPCC: N An Ensembled Framework for the Prediction of the Onset of Diabetes", 4 IEEE International conference on Signal Processing, computing and control (ISPCC2k17), Sep-21-23, 2017, Solan, India, 978-15090-5838-9/17\$31@2017 IEEE.

12. EhsanPashaee, Abdullah ChaleChale, "Predicting Diabetes Symptoms by Means of Data Mining Techniques: Study Conducted in Kermanshah Iran", International Academic Journal of Science and EngineeringVol. 3, No. 5, 2014, pp. 1122.ISSN 2454-389611.

13. Ravi Sanakal, Smt. T Jayakumari, "Prognosis of Diabetes Using Data mining Approach-Fuzzy C Means Clustering and Support Vector Machine", International Journal of Computer Trends and
Technology (IJCTT) - volume 11 number 2 May 2014.

14. K. Rajesh, V. Sangeetha," Application of Data Mining Methods and Techniques for Diabetes Diagnosis", International Journal of Engineering and Innovative Technology (IJEIT) Volume 2, Issue 3, September 2012, ISSN: 2277-3754.

15. R. Brindha, P. Anitha,"A Classification And Prediction Model For Diabetic Dataset By Using Different Transformation Techniques", International Journal of Contemporary Research in Computer Science and Technology (IJCRCST) ISSN: 2395-5325 Volume 3, Special Issue 3 (September'2017).

16. G. Krishnaveni*, Prof. T. Sudha," A Novel Technique To Predict Diabetic Disease Using Data Mining - Classification Techniques", International Journal of Advanced Scientific Technologies, Engineering and Management Sciences (IJASTEMS-ISSN: 2454-356X) Volume.3, Special Issue.1, March.2017.

17. VrushaliBalpande, RakhiWajgi," Review on Prediction of Diabetes using Data Mining Technique", International Journal of Research and Scientific Innovation (IJRSI) | Volume IV, Issue IA, January 2017 | ISSN 2321-2705.

18. Harleen, Dr. Pankaj Bhambri,"A Prediction Technique in Data Mining for Diabetes Mellitus", Harleen \& Bhambri, Apeejay-Journal of Management Sciences and Technology, 4 (1), October-2016 ISSN -2347-5005.

19. T. monika Singh, Rajashekarshastry, "Prediction of Diabetes Using Probability Approach", International Research Journal of Engineering and Technology(IRJET) e-ISSN: 2395 -0056 Volume: 04 Issue: 02 | Feb -2017www.irjet.net p-ISSN: 2395-0072.

20. Divya Sharma, Anand Sharma, Vibhakar Mansotra,"A Literature Survey on Data Mining Techniques to Predict Lifestyle Diseases" International Journal for Research in Applied Science \& Engineering Technology (IJRASET), Volume 5 Issue VI, June 2017 IC Value: 45.98 ISSN: 2321-9653. 